\title{
Evaluation of a Vibrotactile Feedback Device for Spatial Guidance
}

\author{
Bernhard Weber ${ }^{1}$, Simon Schätzle ${ }^{2}$, Thomas Hulin ${ }^{2}$, Carsten Preusche ${ }^{2}$ and Barbara Deml ${ }^{1}$ \\ ${ }^{1}$ University of Magdeburg; \\ Institute of Ergonomics, Manufacturing Systems and \\ ${ }^{2}$ German Aerospace Center (DLR); \\ Automation, Germany \\ Institute of Robotics and Mechatronics, Germany
}

\begin{abstract}
In the present study, a vibrotactile feedback device for spatial guidance was evaluated in a tracking task paradigm. Participants $(N=18)$ had to translate and rotate virtual objects according to the vibrotactile vs. verbal cues without visual information.

Both types of spatial guidance were evaluated using objective performance data (i.e. speed, accuracy) as well as subjective judgments. Results indicate that distinguishing spatial cues during the translational task was more difficult when being guided by vibrotactile feedback compared to verbal feedback. Nevertheless, individuals with vibrotactile guidance showed better performance at rotational tasks. Implications for the further design process and other areas of application are discussed.
\end{abstract}

KeYwords: Haptic I/O, Voice I/O, Human Factors.

INDEX TERMS: H.5.2 [Information Interfaces and Presentation] User Interfaces---Haptic I/O, Evaluation/methodology; General Terms: Human Factors, Experimentation.

\section{INTRODUCTION}

During the last years, vibrotactile displays have not only been used to support visually impaired users, but there are more and more applications, in which these devices are also applied for assisting sighted persons. Particularly, the attempt of enhancing the spatial awareness of human operators has gained a lot of attention. This is mainly due to the following reasons [1]: 1) Usually, operators have to process a lot of visual and acoustical information in complex working scenarios. In contrast, the tactile channel is less overloaded. 2) Vibrotactile stimulation allows for displaying information in an unobtrusive way without annoying others or drawing their attention to confidential matters. 3) Finally, as the stimuli are directly mapped to body coordinates, it is also a quite intuitive form of feedback (e.g. the user simply follows the direction of vibrotactile stimulation).

The paper is structured as follows: First, a short literature overview of vibrotactile guidance is provided (Sec. 2) and the vibrotactile feedback device "VibroTac" is introduced (Sec. 3). VibroTac was evaluated concerning its capability of guiding human operators towards a specific spatial target. Within this work, both the experimental procedure and the results are presented (Sec. 4). Next, the outcome of the study is discussed (Sec. 5) and summarized in a concluding remark (Sec. 6).

\{Bernhard.Weber, Barbara.Deml\}@ovgu.de,

\{Simon.Schaetzle, Thomas.Hulin, Carsten.Preusche\}@dlr.de

IEEE World Haptics Conference 2011

21-24 June, Istanbul, Turkey

978-1-4577-0297-6/11/\$26.00 @2011 IEEE

\section{Previous Research on Vibrotactile Guidance}

Vibrotactile feedback has been provided to various parts of the body successfully: In some studies a belt is worn around the abdomen [2]. Others use shoulder pads [3] or apply vests to stimulate the ventral or dorsal torso [4]. Just the same, the wrist or the forearm is actuated by arm bands [5]. Besides, tactors have also been applied to the fingertip and the hand [6]. Within some studies even larger areas of the body are actuated by a wearable data suit [7].

Besides, studying the stimulation of different body parts, vibrotactile feedback has also been used for a variety of applications and purposes (e.g. sensory substitution, communication of complex messages; for an overview see [1]). Regarding vibrotactile guidance, three different areas can be distinguished:

a) Attentional guidance. Vibrotactile warning signals can be highly effective in directing visual attention to the location of critical events. This was shown in the context of car driving [8] and air traffic control [9].

b) Movement guidance. Vibrotactile feedback has also been applied for guiding arm movements and thus, for enhancing motor learning and training. Such, for instance, it was helpful for the teaching of good posture and bowing technique to novice violin players [10].

c) Spatial guidance. Furthermore, vibrotactile stimulation has been used effectively in order to guide human operators towards a specific target. This feedback has proven to be helpful for surgeons, when complex trajectories have to be followed [1]. It was also found to support marksmanship [11] or to aid dismounted soldiers in navigating through unfamiliar environments [12]. Vibrotactile devices were also used in order to guide actors in a virtual studio and to help them in interacting with virtual objects [13]. Besides, navigating vehicles, such as helicopters or boats, through a series of waypoints can be supported by vibrotactile devices [14]. Finally, there are further scenarios that all have in common that the spatial frame of reference is disturbed, such as in maritime scenarios [15], in weightless environments in space [16], in the cockpit of an aircraft [17], in virtual environments [18] or in patients suffering of balance disorders [19].

This work is dedicated to the paradigm of spatial guidance. Thereby, one major challenge is to determine how to convey distance and direction information. There are some studies, in which no meaningful effect on performance (i.e. speed, accuracy) could have been detected when adding distance information [20] [21]. For this reason, it seems to be more interesting to concentrate on the encoding of direction. Particularly, the number and the optimal configuration of tactors still need further research. Thus, our first research question addresses whether a higher degree of spatial resolution increases the user's performance or not. Similarly, it is still unclear how well vibrotactile feedback is actually suited for providing spatial guidance. This question can 
only be answered when contrasting it to another feedback modality. Within the context of navigation, verbal guidance has shown to be effective [22] [23]. Thus, our second research question focuses on the direct comparison of both modalities.

\section{The Vibrotactile Feedback DeVice Vibrotac}

VibroTac is a vibrotactile feedback device which was developed at the German Aerospace Center (DLR). It is used to apply vibrotactile stimuli to the human arm [24].

In previous psychophysical studies, the alignment, number and type of vibration motors of a vibrotactile wrist band have been investigated [25]. Experiments to determine the spatial acuity revealed that a configuration with six actuators distributed on the arm's perimeter in equal distances is a good compromise between the number of feedback locations and reliable detection of feedback locations when compared with configurations of four and eight actuators [25].

Due to the ergonomic concept of the device, it can be worn on a wide range of arm diameters while battery power and a wireless control interface contribute to unrestricted movement capability and user convenience.

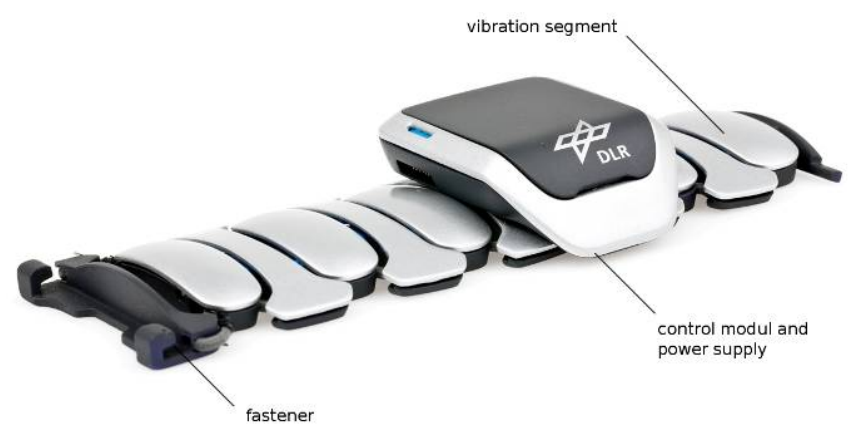

Figure 1. The vibrotactile feedback device "VibroTac"

Six vibration segments are distributed around the human arm in equal distances. Each segment comprises a cylindrical DC vibration motor which is separately and continuously adjustable in frequency $(0-190 \mathrm{~Hz})$ and shape of stimuli (e.g. impulse, ramp, wave, knock, trembling).

Up to 64 devices can be controlled simultaneously. This allows the use of several devices worn on arms or even on legs for distributed feedback. The maximum data update rate is $1600 \mathrm{~Hz}$ (if several devices are used, the maximum update rate is divided through the number of devices). The time constant of the motors itself is approximately $50 \mathrm{~ms}$.

As several persons use the device, hygienic material (plastic) is used and skin-touching areas are designed to be small in order to avoid sweating.

\section{Evaluation EXPERIMENT}

In a laboratory experiment, we compared vibrotactile guidance provided by VibroTac and verbal guidance in a tracking task paradigm. Moreover, we compared vibrotactile guidance with six and four directional cues to explore whether a higher degree of spatial resolution results in better performance.

\subsection{Methods}

\section{Sample}

$N=18$ ( 1 female, 17 male) right-handed individuals (employees of the German Aerospace Center; $M_{\mathrm{AGE}}=28.2$ years; $S D=5.2$ with an age range from 22 to 43 years) were recruited; none of them had participated in prior studies on VibroTac.
Assessment of Motor Skills

Participants' fine motor skills were assessed with the motor performance test of the Vienna Test System (Schuhfried GmbH). Based on Fleishman's taxonomy [26], the precision of arm-hand movements ("control precision") was measured in a track tracing test. Although abilities were distributed normally (KolmogorovSmirnov-Z $=0.85 ; n s)$, the mean percentile rank of $M=68.4(S D=$ 28.2, Range $=24-100$ ) indicates above-average abilities of the recruited sample.

Psychophysical Adjustment of Modalities

To guarantee that the perceived stimulus magnitudes are similar for both guidance modes, we matched the intensities of the vibrotactile and the acoustical stimuli using the cross modality matching method developed by Stevens [27]. $N=10$ individuals (who did not participate in the main study) adjusted the stimulus intensities in one modality to a series of five reference stimuli in the other modality until they were perceived as equally strong. In a similar manner, the other modality had to be adjusted afterwards. The matching order was randomized for each participant.

As can be seen in Figure 2, the results of both matching orders were similar.

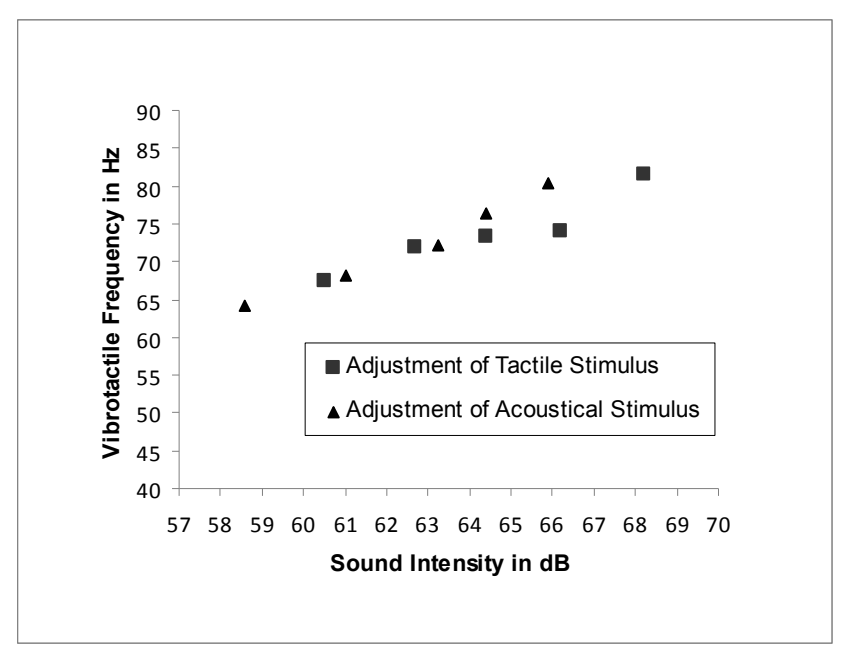

Figure 2. Results of the cross modality matching

For the main experiment, we chose a moderate sound intensity of $63 \mathrm{~dB}$ and the corresponding vibrotactile frequency of $73 \mathrm{~Hz}$.

Comparison of Vibrotactile and Verbal Guidance

Experimental Task. Participants had to move their right arm to specific target positions. Subsequently, they had to turn their right hand until a specific rotation angle was reached. After each trial, participants moved their hand back to the middle of their working space and the next trial was started. Participants were allowed to have a short rest before starting the next trial to avoid physical exhaustion.

Experimental Design. Two types of vibrotactile guidance were compared: vibrotactile guidance with four directional cues and with six directional cues. In the condition with verbal guidance, four directional cues ("up", “down", "left", "right") were provided.

A within-subjects design is utilized with the type of guidance as within factor. The order of the three experimental conditions (vibrotactile with six vs. four directions vs. verbal guidance) was permuted to control for potential order or time effects (fatigue, learning, etc.), resulting in six condition orders (factorial of 3 ). Each participant was assigned to one of the six experimental conditions. For each condition we assigned an individual with 
high, moderate and low motor skills (precision of arm/ hand movement).

Procedure. Participants took a seat and read instructions on the experimental task. Subjects were told that they would start each run from the same starting position (in the middle of the working space) with their right arm extended forward. Then they were asked to follow the translational cues until the target positions were reached and turn their hand/ arm in the indicated direction until the pre-defined angles were reached. The virtual targets were placed in a vertical plane in front of the participants and could be reached easily with the extended arm. Furthermore, participants were instructed not to rotate their arm during the translational task, i.e. the reference frame of spatial cues in the conditions with VibroTac was always the same. Participants were instructed to work as accurately and as quickly as possible.

In the condition "Vibrotactile guidance with six directions" (henceforth labeled as "VT6") individuals were informed that they would wear VibroTac on the wrist of their right arm. Each of the six actuators will indicate a specific direction, i.e., the arm should be moved in the direction of the activated motor ("pull metaphor"). When reaching the target position, all actuators will be activated by two short impulses. For the rotation task, the actuators will be activated clockwise ("turn right") or anticlockwise ("turn left"). When reaching the target angle, all actuators will be de-activated and re-activated (in the opposite direction) when overshooting the target angle.

In the "Vibrotactile guidance with four directions" (VT4) condition, individuals were informed that there were four translational cues ("up", “down", "left", "right"). The rotational cues were the same as in condition VT6.

Finally, in the "Verbal guidance" (VB) condition, we instructed participants, that there were four verbal commands ("top", "bottom", "left", "right") for the translational task. Once they have reached the target position, the direction of rotation was indicated by the commands "left" or "right".

After instruction, participants were asked to put on the headphones for the verbal instructions and a blindfold, to avoid any visual distraction (see Figure 3). Prior to each main trial, individuals were familiarized with the corresponding guidance mode and they practiced on both the translational and the rotational task in three exercise trials. Then, the main trial with ten tasks was started. All target positions were chosen randomly, but identical for each trial and participant.

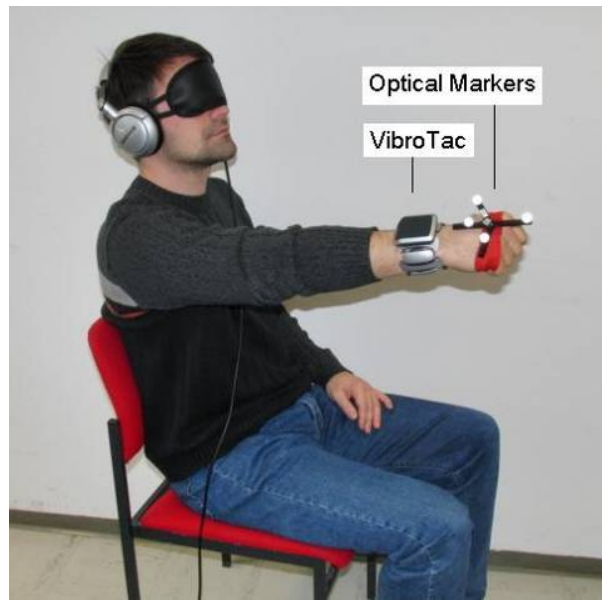

Figure 3. Experimental setup

In all conditions, the hand movements were tracked with an optical tracking system (VICON). Vibrotactile as well as verbal cues were generated automatically and modified contingent on the current arm position.
The verbal cues were recorded prior to the experiment and were identical concerning sound intensity and duration $(0.7 \mathrm{sec})$.

In the translational task, spatial cues were provided every 0.7 sec; stimulus duration in both the vibrotactile and the verbal condition was $0.7 \mathrm{sec}$. For the rotational task, the vibrotactile cues for "left" and "right" were realized by a (anti-) clockwise activation of the tactors without interruptions.

After each trial, participants filled out the NASA-TLX questionnaire [28] in order to measure perceived workload and a questionnaire to evaluate the corresponding guidance mode.

After having finished all three experimental trials, participants were asked to complete a post-experimental questionnaire with an overall assessment of all guidance modes.

\subsection{Results of the Evaluation Experiment}

For the evaluation of three different guidance modes, objective performance data as well as subjective rating data were gathered.

\section{Performance Data}

Time to Complete Translation. As a first performance measure, the average time required to reach the target positions was determined. Repeated measures analysis of variance (ANOVA) with the experimental condition as within factor revealed a marginally significant effect $(F(2,34)=3.63 ; p=.05 ; \eta 2=.18)$. Subsequent post-hoc contrasts with Bonferroni adjustment showed that participants in the VB condition reached the target positions significantly faster $(M=6.2 \mathrm{~s} ; S D=1.3)$ compared to VT4 condition $\left(M=8.1 \mathrm{~s} ; S D=2.3 ; p_{\text {VB-VT4 }}<.01\right)$. Yet, contrasting the VT6 $(M=7.9 \mathrm{~s} ; S D=3.5)$ with the remaining conditions did not yield significant differences. In Figure 4, the corresponding means and $95 \%$ confidence intervals are depicted.

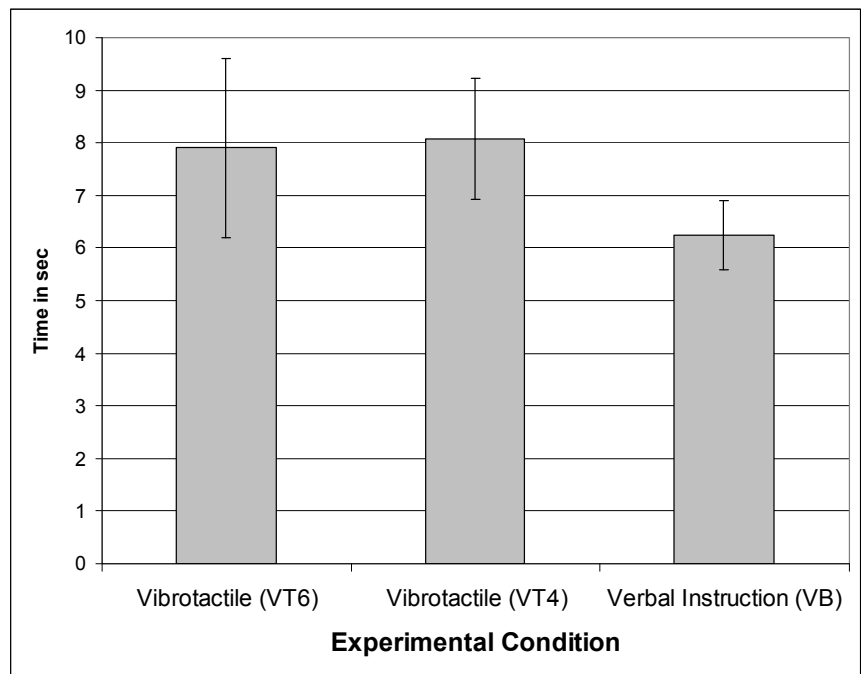

Figure 4. Time to complete translation

Time to Complete Rotation. Next, the corresponding time for rotation was analyzed. Yet, two cases had to be excluded from the following analyses due to technical problems that occurred during the rotation task (interruption of hand tracking or data transmission, respectively). The results from both vibrotactile conditions (VT6, VT4) were averaged, because the rotational cue pattern was identical in these conditions. A highly significant difference was found when comparing the average rotation times in the VT conditions $(M=5.9 \mathrm{~s} ; S D=1.2)$ and the VB condition $(M=7.1 \mathrm{~s} ; S D=0.77 ; t(15)=4.2 ; p=.001$, see Figure 5$)$. 


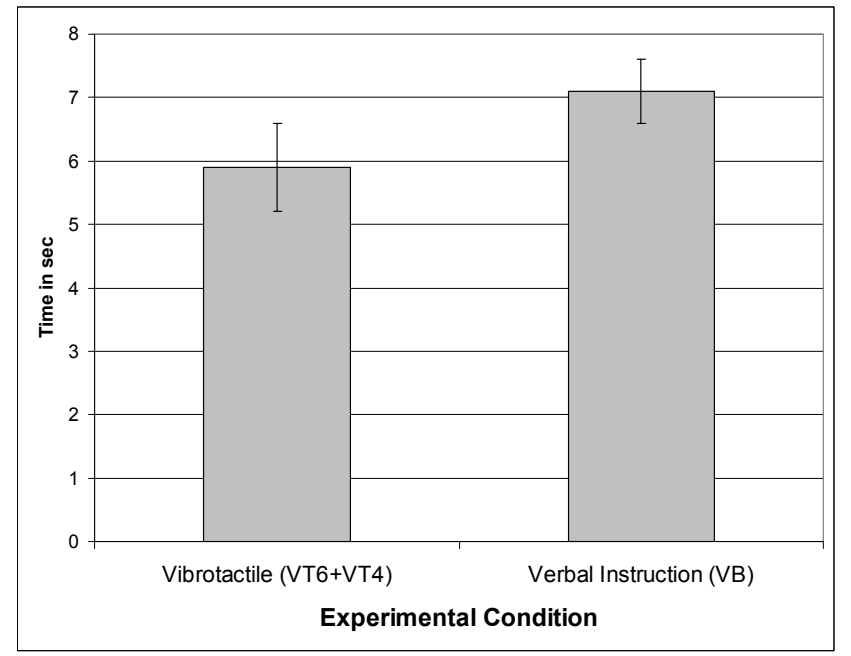

Figure 5. Time to complete rotation

Translational Accuracy. As a measure for translational accuracy, the ratio of the actual path length and the direct path from the corresponding starting point to the target position was determined. Thus, higher values indicate less accurate movements.

Although the ratio was lowest in the VT6 condition $(M=1.9$; $S D=.40)$ and somewhat higher in the VT4 $(M=2.1 ; S D=.38)$ and VB condition $(M=2.0 ; S D=.44)$, the conventional level of significance was not reached in a repeated measures ANOVA $(F$ $(2,34)=1.49$; ns. $)$.

Rotational Accuracy. The sum of all rotation angles until reaching the target was divided by the sum of rotation angles required to reach the target position in all trials. Consequently, higher values indicate lower accuracy. Again, two cases were excluded. The corresponding value for the VT conditions was $M=$ $6.2(S D=2.8)$ and $M=7.5(S D=4.4)$ in the VB condition. Yet, the difference did not reach significance $(t(15)=1.2 ; n s$. $)$ due to the large amount of variance in both conditions.

As an additional criterion for rotational accuracy, we measured the maximum angle when overshooting the target position. Comparing the average overshooting revealed that participants were more accurate in the VT conditions $\left(M=17.5^{\circ} ; S D=10.9\right)$ than in the VB condition $\left(M=24.1^{\circ} ; S D=13\right)$, although the difference did not reach significance $\left(p_{\mathrm{VT}-\mathrm{VB}}=.11\right.$, two-tailed testing).

\section{Subjective Data}

Workload. The NASA-TLX questionnaire with six dimensions (cognitive demands, physical demands, temporal demands, performance, effort, and frustration) was administered (20-point Likert scales). Moreover, the relative importance of the six dimensions was rated in paired comparisons at the end of the experimental session and a weighted average workload score was computed.

Comparing the overall workload scores in a repeated measures ANOVA revealed significant differences between the experimental conditions $\left(F(2,34)=4.87 ; p<.05 ; \eta^{2}=.22\right)$. Conducting a post-hoc contrast analysis (with Bonferroni adjustment) showed that the workload judgments in the VB condition $(M=6.8$ on a scale ranging from $1-20 ; S D=.46)$ were significantly lower than the corresponding ratings in the VT4 condition $\left(M=9.0 ; S D=.76 ; p_{\mathrm{VB}-\mathrm{VT} 4}<.05\right)$. Contrasting VT6 $(M$ $=7.8 ; S D=.67)$ with the remaining conditions did not yield significance. The overall result pattern, with the highest workload ratings in the VT4, followed by VT6 and the lowest ratings in the VB condition was found for all six NASA-TLX dimensions.

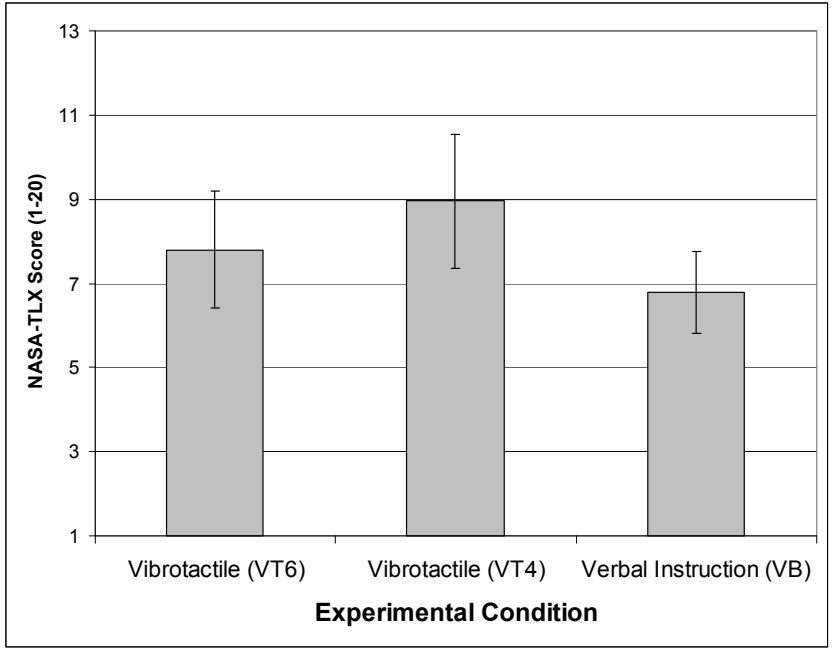

Figure 6. NASA-TLX overall workload ratings

Cue Frequency. Individuals indicated whether it would have been helpful to provide directional cues more frequently in the corresponding conditions (1= "strongly disagree"; $7=$ "strongly agree"). A one-way ANOVA revealed significant differences between conditions $\left(F(2,34)=6.1 ; p<.05 ; \eta^{2}=.27\right)$. Specifically, moderate values in the VB condition $(M=4.0 ; S D=$ $2.1)$ indicate that cue frequency was not sufficiently high. The values in the VT6 $(M=2.7 ; S D=1.6)$ and the VT4 condition $(M$ $=2.7 ; S D=1.4)$ tended to be lower in the former case $(p<.10)$ or were significantly lower in the latter case $(p<.05)$.

Appropriateness for Spatial Guidance. Participants indicated whether the three different guidance modes were appropriate for spatial guidance (scale ranging from $1=$ "not at all" to $7=$ "very appropriate").

While the corresponding ratings in the VB condition $(M=5.7$; $S D=1.4)$ and the VT6 condition $(M=5.4 ; S D=1.2)$ were relatively high, ratings were lower in the VT4 condition $(M=4.4$; $S D=1.2$ ). Accordingly, ANOVA yielded a significant effect of experimental condition $\left(F(2,34)=5.0 ; p=.01 ; \eta^{2}=.23\right)$. Indeed, the ratings in the VT4 condition were significantly lower compared to the VB $(p<.05)$ or tended to be lower compared to the VT6 condition $(p<.07)$.

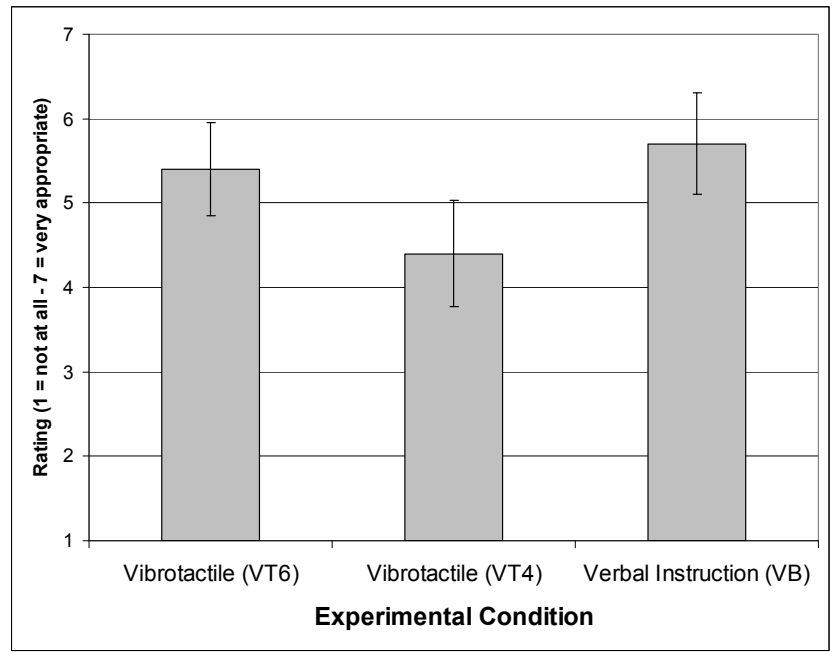

Figure 7. Appropriateness for spatial guidance 
Additional Distance Coding. In the current study, no distance information was encoded. Yet, participants indicated that additional distance information (e.g., by varying signal intervals or signal intensity) would have been helpful for the translational $(M=4.9 ; S D=2.1 ; 1=$ not at all; $7=$ very important $)$ as well as for the rotational task $(M=4.6 ; S D=2.1)$.

\section{Discussion}

In the present study, we investigated several types of spatial guidance in a simple tracking task paradigm. Specifically, we compared vibrotactile guidance with four vs. six feedback directions and a verbal guidance mode with four feedback directions. Participants had to move their arm to target positions and turn their hand until a certain angle was reached without having any visual feedback. Moreover, individuals were instructed to work as quickly as possible to trigger time pressure.

We evaluated the different guidance modes by comparing completion times, movement accuracy, and subjective ratings.

Regarding the translational task, we found evidence that individuals are fastest when being supported by verbal guidance, although no significant difference between verbal and vibrotactile guidance with six feedback directions was evident.

One explanation for the lower performance in the vibrotactile guidance conditions is the difficulty to distinguish the translational cues as reported by participants. Despite the fact that individuals needed more time to detect and interpret the vibrotactile cues, the movement accuracy was similar for vibrotactile and verbal guidance.

While verbally instructed participants required less time to complete the translational task, the opposite was true for the rotational task. Individuals with vibrotactile guidance reached the target angles faster and more accurately than in the verbal guidance condition.

This positive effect of vibrotactile guidance can be explained by the fact that the distinction of spatial information (clockwise vs. anti-clockwise activation of VibroTac) was much easier when performing the rotational task. Moreover, spatial information was provided continuously, i.e., information density was higher compared to verbal instruction. Consistently, individuals indicated that cue frequency was too low in the verbal guidance condition.

Subjective ratings also indicated that workload was highest when working with only four vibrotactile feedback directions. The higher workload in this condition might be due to the lower degree of movement accuracy.

Accordingly, individuals rated this guidance mode as least appropriate for spatial guidance, while both vibrotactile guidance with six directions and verbal guidance were rated as relatively supportive and helpful.

\section{ConcLusion AND OUTLOOK}

In this paper a study is presented evaluating the use of VibroTac attached to the human wrist for guiding subjects on a predefined trajectory. The appropriateness of the vibrotactile stimulus was proven comparing it with verbal instructions as they are often used in daily life. Individuals achieved similar performance for the translational and better performance for the rotational subtask when using the six actuators of VibroTac. Thus, it could be stated that its use is valuable, especially in environments in which the verbal guidance is not useful, like in noisy environments.

Despite the positive overall assessment of VibroTac, the provided feedback could be improved by adding distance information or by further reducing the ambiguity of vibrotactile stimuli. This leads to further research on how to exploit this powerful tool.

Based on the results of the current study, future research will be conducted on how vibrotactile feedback supports the skill acquisition for motor tasks and for which domains vibrotactile stimuli can be used for motion guidance, attention direction or display of geometric information (collision feedback).

\section{REFERENCES}

[1] L. A. Jones and N. B. Sarter, "Tactile displays: Guidance for their design and application", Human Factors, 50 (1), pp. 90-111, 2008.

[2] J. Bluteau, M.-D. Dubois, S. Coquillart, E. Gentaz, and Y. Payan, "Vibrotactile guidance for trajectory following in computer aided surgery", IEEE Eng. in Med. and Bio., pp. 2085-2088, Aug/Sept. 2010.

[3] A. Toney, L. Dunne, B. H. Thomas, and S. P. Ashdown, "A shoulder pad insert vibrotactile display”, IEEE ISWC, pp. 35-44., Oct. 2003.

[4] Z. Q. Ding, I. M. Chen, and S. H. Yeo, "The development of a realtime wearable motion replication platform with spatial sensing and tactile feedback", IEEE IROS, pp. 3919-3924, Oct. 2010.

[5] J. Y., Ng, and J. Man, "Vibro-Monitor: A vibrotactile display for physiological data monitoring" Conf. on Human Interface Technologies, Dec. 2004.

[6] M. Brell, "Eine vibrotaktile Mensch-Maschine-Schnittstelle fuer chirurgische Applikationen" [A vibrotactile human-machineinterface for surgical applications], $\mathrm{PhD}$ thesis, University of Oldenburg, 2009.

[7] T. McDaniel, D. Villanueva, S. Krishna, and S. Panchanathan, "MOVeMENT: A Framework for systematically mapping vibrotactile stimulations to fundamental body movements". IEEE Have, pp. 1-6, Oct. 2010.

[8] C. Ho, H. Z. Tan, and C. Spence, "Using spatial vibrotactile cues to direct visual attention in driving scenes", Transportation Research, Part F: Traffic Psychology and Behaviour, 8(6), pp. 397-412, 2005.

[9] B. Weber, B. Deml, M. Friedrich, S. Schätzle, H. Oberheid, and C. Preusche, "Vibrotaktiles Feedback zur Aufmerksamkeitslenkung bei komplexen Lotsentätigkeiten. [Vibrotactile feedback for attention direction in a complex air traffic controlling task]. In M. Grandt \& A. Bauch (Eds.): Innovative Interaktionstechnologien für MenschMaschine-Schnittstellen, pp. 71-85, 2010.

[10] J. van der Linden, W. Schoonderwaldt, and J. Bird, "Good vibrations: guiding body movements with vibrotactile feedback", Proceedings of 3rd Int. Workshop on Physicality, Sept. 2009.

[11] T. Oron-Gilad, J. L. Downs, R. D. Gilson, and P. A. Hancock, "Vibrotactile guidance cues for target acquisition", IEEE Transactions on Systems, Man, and Cybernetics, Part C: Applications and Reviews, 37(5), pp. 993-1004, 2007.

[12] L. R. Elliott, J B. F. van Erp, E. S. Redden, and M. Duistermaat, „Field-based validation of a tactile navigation device”, IEEE Transactions on Haptics, 3(2), pp. 78-87, 2010.

[13] B. Wöldecke, T. Vierjahn, M. Flasko, J. Herder, and C. Geiger, "Steering actors through a virtual set employing vibro-tactile feedback", TEI, pp. 169-174, Feb. 2009.

[14] R. Tilak, I. Xholi, D. Schowalter, et al., "Crossmodal links in attention in the driving environment: The roles of cueing modality, signal timing, and workload", Proc. of the Human Factors and Ergonomics Society, pp. 1815-1819, Sept. 2008.

[15] J. B. F. van Erp, and B. P. Self, "Introduction to tactile displays in military environments". In Tactile Displays for Orientation, Navigation and Communication in Air, Sea, and Land Environments, NATO RTO meeting, Nueilly-sur-Seine, 2008.

[16] R. Traylor, and H. Tan, "Development of a wearable haptic display for situation awareness in altered-gravity environment: Some initial findings", IEEE Haptics, pp. 159-164, March, 2002.

[17] A. Rupert "An instrumentation solution for reducing spatial disorientation mishaps", IEEE Eng. in Med. and Bio., pp. 71-80, March/April 2000.

[18] J. B. F. van Erp, "Tactile Displays in Virtual Environments". In What is essential for virtual environments to meet military training goals? NATO RTO meeting, Nueilly-sur-Seine, 2001.

[19] E. Piateski and L. Jones, "Vibrotactile pattern recognition on the arm and torso", IEEE WHC: pp. 90-95, March 2005. 
[20] L. A. Jones, B. Lockyer, and E. Piateski, "Tactile display and vibrotactile recognition on the torso", Advanced Robotics, 20(12), pp. 1359-1374, 2006.

[21] M. Straub, A. Riener, and A. Ferscha, "Distance encoding in vibrotactile guidance cues", IEEE, MobiQuitous, pp. 1-2, July 2009.

[22] R. L. Klatzky, N. A. Giudice, J. R. Marston, et al., "An n-back task using vibrotactile stimulation with comparison to an auditory analogue", Behav. Res. Methods, 40(1), pp. 367-372, 2008.

[23] N. A. Giudice, J. Z. Bakdash, G. E. Legge, and R. Roy, "Spatial learning and navigation using a virtual verbal display", $A C M$ Transactions on Applied Perception, 7(1), pp. 1-22 (Article 3), 2010.

[24] S. Schätzle, T. Ende, T. Wuesthoff, and C. Preusche, "VibroTac: An ergonomic and versatile usable vibrotactile feedback device", IEEE International Symposium in Robot and Human Interactive Communication (Ro-Man), Viareggio, Italy, Sep. 2010.

[25] S. Schätzle, T. Hulin, C. Preusche, and G. Hirzinger, "Evalution of vibro-tactile feedback to the human arm", Proceedings of Eurohaptics, pp. 557-560, Paris, France, 2006.

[26] J. B. F. Fleishman, "Structure and measurement of psychomotor abilities". In The Psychomotor Domain, R.N. Singer, pp. 78-196. Philadelphia: Lea \& Febiger, 1972.

[27] S. S. Stevens, "Cross-modality validation of subjective scales for loudness, vibration, and electric shock", Journal of Experimental Psychology, 57(4), pp. 201-209, 1959.

[28] S. G. Hart and L. E. Staveland, "Development of NASA-TLX (Task Load Index): Results of empirical and theoretical research". In Human Mental Workload, P.A. Hancock \& N. Meshkati, NorthHolland Press, Amsterdam, 1988. 